Advances in Fuzzy Mathematics.

ISSN 0973-533X Volume 12, Number 2 (2017), pp. 319-332

(C) Research India Publications

https://dx.doi.org/10.37622/AFM/12.2.2017.319-332

\title{
Fuzzy Reliability Analysis of a Summer Air Conditioning System
}

\author{
M. K. Sharma \\ Department of Mathematics, \\ R.S.S. (PG), Pilkhuwa, (Hapur) U.P. - India-245304
}

\begin{abstract}
In this paper, we have considered a summer air conditioning system for its reliability and fuzzy reliability analysis. Fuzzy Reliability and mean time to fuzzy failure as well as reliability and mean time to failure for considered system have been computed with the help of fuzzy operators which is the expansion of Boolean function algorithm. The considered system works as air conditioned for a place in hot and dry weather like Nagpur, Bhopal and Delhi etc. The comfort conditions required in an air conditioned space are $26^{\circ} \mathrm{C}$ dry bulb temperature and $63 \%$ (relative humidity).
\end{abstract}

Key words: Fuzzy sets, Reliability, Failure probability, Fuzzy number, Mean time to fuzzy failure (MMTFF), Air Conditioning System.

\section{AMS Subject classification: 68M15}

\section{INTRODUCTION:}

It is well known that the system reliability evaluation for a complex system is based on the analysis of failure rates of its component or subsystems and their effect on the system failure. In classical reliability analysis, the uncertainties in the failure probability and / or failure rate of system components or basic components can be propagated to find the uncertainty in the overall system failure probability [3]. The present paper deals with a comparative study of probabilities and fuzzy set theoretic approach for reliability evaluation. Hence, fuzzy sets, which were developed by L. A. Zadeh [7], thirty years ago, can help to overcome this situation. Experts utilize fuzzy sets [2] to subjective describe the uncertainties of each given event failure rate and then perform mathematical operation to evaluate system reliability. 
There are different methods of analyzing the failure behavior of a system. But, in this era of computerization a variety of machines is being automated day by day. The automation of machine enhances its complexity. Also we have many systems of great importance, the system of that type are never expected to be failed eve in testing mode.

In the considered system, two air dampers $A D_{1}$ and $A D_{2}$; two air filters $A F_{1}$ and $A F_{2}$ are working in parallel redundancy to improve system's overall performance. The input air passes through air dampers, air filters, cooling coils, adiabatic humidifier and water eliminator, and then gives output air. The object of the system is to supply cooled air for selected place. It has been assumed that the failure rates of various components of considered system follow arbitrary distribution and there is no repair facility available for a failed component. The arrangement of equipments required for an ordinary system has been represented in fig-1. The block diagram of the system under considerations has been shown in fig- 2 .

Reliability of the complex system has been computed in case of fuzzy failure rates follow either Weibull or exponential time distribution. An important fuzzy reliability parameter, viz; mean time to fuzzy failure (M.T.T.F.F.) has also been computed for considered system. A numerical example together with its graphical representation has been appended in last to highlight important results of the study.

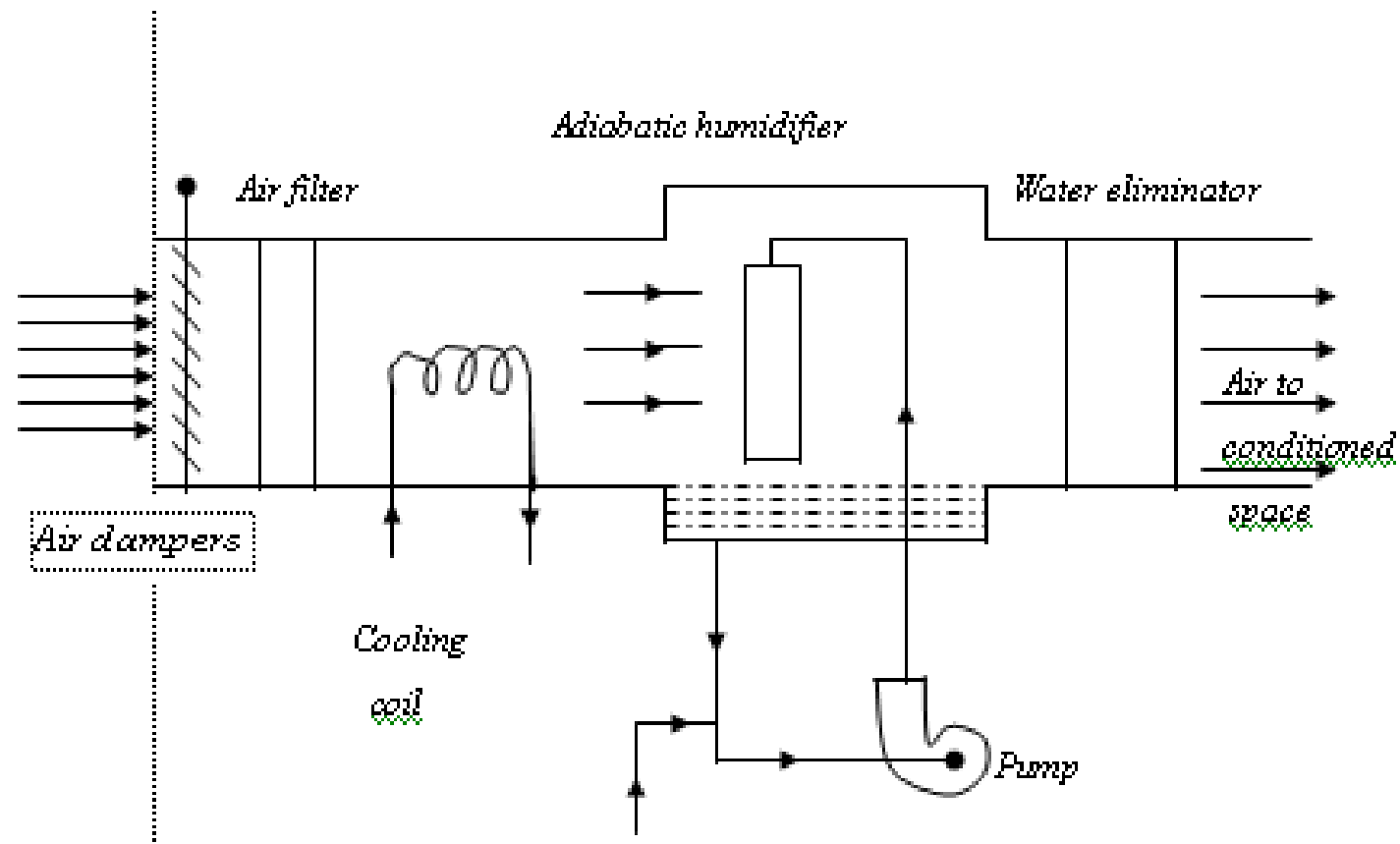

Compensating water

Fig-1: Air conditioning system for hot and dry weather 


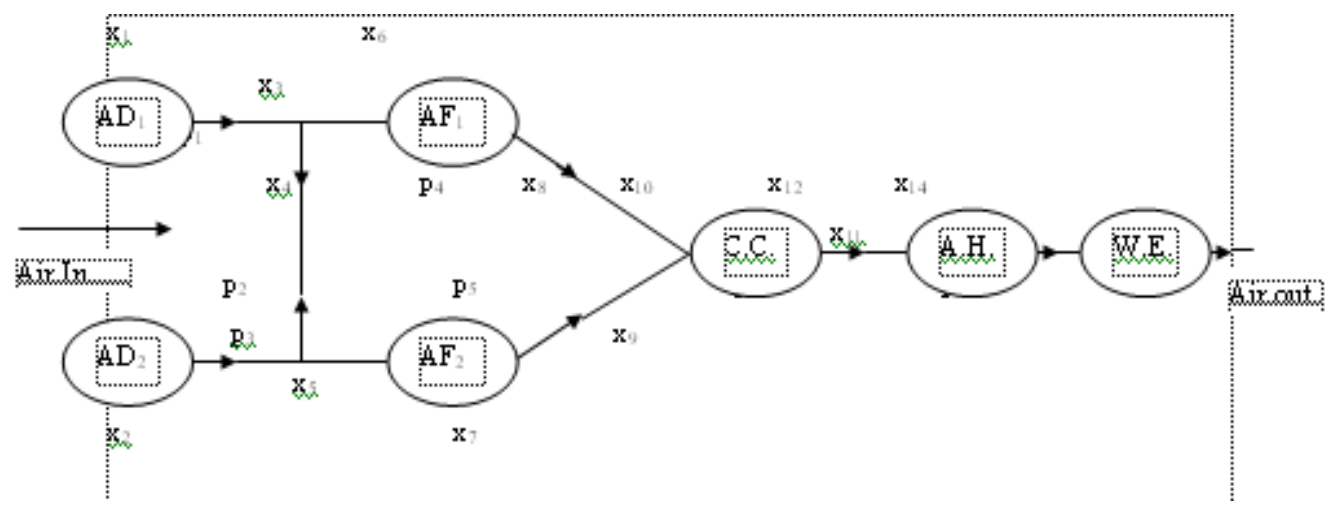

Figure 2: Block-diagram

\section{NOTATIONS:}

Notations used in this model are as follows:

$x_{1}, x_{2}$

$x_{3}, x_{4}, x_{5}$

$x_{6}, x_{7}$

$x_{10}, x_{12}, x_{14}$

$x_{8}, x_{9}, x_{11}, x_{13}$

$\wedge / \vee$

ANF

ORF

$\tilde{\lambda}$

$\tilde{\mu}$

$\mu_{S}\left(s_{i}\right)$

$\cap / \cup$

$x_{i}^{\prime}$

$\tilde{x}_{i}^{\prime}$

$R_{i}$

$\tilde{R}_{i}$

$x_{i}$

$P_{r}(f=1)$

$\tilde{P}_{r}(f=1)$
States of air dampers $A D_{1}, A D_{2}$ respectively.

States of pipes $P_{1}, P_{2}, P_{3}$ respectively.

States of air filters $A F_{1}$ and $A F_{2}$, respectively.

States of cooling coil, air humidifier and water eliminator, respectively.

States of pipes $P_{4}, P_{5}, P_{6}$ and $P_{7}$, respectively.

Conjunction/Disjunction.

Fuzzy Conjunction operator

Fuzzy Disjunction operator

Fuzzy failure rate

Fuzzy repair rate

Membership grade

Intersection/Union.

Negation of $x_{i}, \forall i$

Fuzzy Negation of $x_{i}, \forall i$

Reliability of the component corresponding to state $x_{i}$.

Fuzzy Reliability of the component corresponding to state $x_{i}$.

$=1$, in good state; $=0$ in bad state.

Probability of successful operation of the function $f$.

Fuzzy Probability of successful operation of the function $f$.

\section{FORMULATION OF MATHEMATICAL MODEL}

By using Boolean function technique, the conditions of capability of successful operation of the considered system in terms of logical matrix can be expressed as: 


$$
F\left(x_{1}, x_{2}--, x_{14}\right)=\left[\begin{array}{cccccccccc}
x_{1} & x_{3} & x_{6} & x_{8} & x_{10} & x_{11} & x_{12} & x_{13} & x_{14} & \\
x_{1} & x_{3} & x_{4} & x_{7} & x_{9} & x_{10} & x_{11} & x_{12} & x_{13} & x_{14} \\
x_{2} & x_{5} & x_{7} & x_{9} & x_{10} & x_{11} & x_{12} & x_{13} & x_{14} & \\
x_{2} & x_{4} & x_{5} & x_{6} & x_{8} & x_{10} & x_{11} & x_{12} & x_{13} & x_{14}
\end{array}\right]
$$

\section{OPERATIONS ON TRIANGULAR AND TRAPEZOIDAL FUZZY NUMBERS:-}

Let $A=\left(a_{1}, a_{2}, a_{3}\right)$ and $B=\left(b_{1}, b_{2}, b_{3}\right)$ be two triangular fuzzy numbers. Then the fuzzy addition of triangular fuzzy number $A$ and $B$ is defined as

$$
\tilde{A}+\widetilde{B}=\left(a_{1}+b_{1}, a_{2}+b_{2}, a_{3}+b_{3}\right)
$$

Therefore addition of two triangular fuzzy numbers is again a triangular fuzzy number.

Similarly subtraction of two triangular fuzzy numbers is also a triangular fuzzy number that can be given by the following expressions: -

$$
A-B=\left(a_{1}-b_{1}, a_{2}-b_{2}, a_{3}-b_{3}\right)
$$

Multiplication of two triangular fuzzy numbers A and B need not to be a triangular fuzzy number. The membership function for multiplication of two fuzzy numbers

$$
A=\left(a_{1}, a_{2}, a_{3}\right) \quad \text { and } \quad B=\left(b_{1}, b_{2}, b_{3}\right)
$$

may be given by the following expression.

$\mu_{\tilde{A} * \tilde{B}}(x)=\left\{\begin{array}{cc}-D_{1}+\left[D_{1}^{2}+(x-P) / T_{1}\right]^{1 / 2} & P \leq x \leq Q \\ -D_{1}-\left[D_{2}^{2}+(x-R) / U_{1}\right]^{1 / 2} & Q \leq x \leq R \\ 0 & \text { otherwise }\end{array}\right.$

where, $\quad T_{1}=\left(a_{2}-a_{1}\right)\left(b_{2}-b_{1}\right), T_{2}=a_{1}\left(a_{2}-a_{1}\right)+b_{2}\left(b_{2}-b_{1}\right)$,

$$
\begin{aligned}
& U_{1}=\left(a_{2}-a_{1}\right)\left(b_{2}-b_{1}\right), U_{2}=b_{3}\left(a_{2}-a_{1}\right)+a_{3}\left(b_{2}-b_{1}\right), D_{1}=\frac{T_{2}}{2 T_{1}}, D_{2}=-\frac{U_{2}}{2 U_{1}}, \\
& P=a_{1} b_{1}, Q=a_{2} b_{2}, R=a_{3} b_{3}
\end{aligned}
$$

From the above expression it is evident that $A * B$ is not a triangular fuzzy number. However for computational convenience, the second or higher degree terms in $\mu_{\bar{A} . \bar{B}}(x)$ can be ignored to achieve $\mathrm{A} * \mathrm{~B}$ as a triangular fuzzy number $(P, Q, R)$ or $\left(a_{1} b_{1}, a_{2} b_{2}, a_{3} b_{3}\right)$.

The algebraic operations on trapezoidal fuzzy numbers are also defined in a similar manner. Let $A=\left(a_{1}, a_{2}, a_{3}, a_{4}\right)$ and $B=\left(b_{1}, b_{2}, b_{3}, b_{4}\right)$ be two trapezoidal fuzzy numbers. 
Then the addition and subtraction of these two trapezoidal fuzzy numbers is given by trapezoidal fuzzy numbers $\left(a_{1}+b_{1}, a_{2}+b_{2}, a_{3}+b_{3,} a_{4}+b_{4}\right)$ and $\left(a_{1}-b_{1}, a_{2}-b_{2}, a_{3}-b_{3}, a_{4}-b_{4}\right)$ respectively. Again the products of these two trapezoidal fuzzy numbers need not to be trapezoidal. But it can be approximated to $\left(a_{1} b_{1}, a_{2} b_{2}, a_{3} b_{3}, a_{4} b_{4}\right)$.

\section{FUZZY OPERATORS: -}

Now using algebraic operations on fuzzy numbers (triangular or trapezoidal), we can obtain fuzzy operators corresponding to Boolean operators AND, OR etc. Let $\tilde{p}_{1}, \tilde{p}_{2} \ldots \ldots \tilde{p}_{n}$ are the possibility functions of the basic events. Then fuzzy AND and OR operators denoted by ANF and ORF respectively, can be defined as:

$\tilde{p}_{y}=\operatorname{ANF}\left(\tilde{p}_{1}, \tilde{p}_{2}, \ldots \ldots \ldots \ldots \ldots . . . . . . \tilde{p}_{n}\right)=\prod_{i=1}^{n} \tilde{p}_{i}$, where $\prod$ denotes the fuzzy multiplication and $p_{y}$ be the possibility of resulting event.

Let $\tilde{p}_{i}$ 's are represented by triangular fuzzy numbers i.e.

$$
\tilde{p}_{i}=\left(a_{i 1}, a_{i 2}, a_{i 3}\right), \text { where } i=1,2 \ldots n
$$

Then $\tilde{p}_{y}=\operatorname{ANF}\left(\tilde{p}_{1}, \tilde{p}_{2}, \ldots \ldots \ldots \ldots \ldots . . . \tilde{p}_{n}\right)=\left(\prod_{i=1}^{n} a_{i 1}, \prod_{i=1}^{n} a_{i 2}, \prod_{i=1}^{n} a_{i 3}\right),=\left(a_{y 1}, a_{y 2}, a_{y 3}\right)$, say

$$
\begin{aligned}
\tilde{p}_{y} & =\operatorname{ORF}\left(\tilde{p}_{1}, \tilde{p}_{2}, \ldots \ldots \ldots \ldots \ldots . . . \tilde{p}_{n}\right)=1-\prod_{i=1}^{n} 1-\tilde{p}_{i}=1-\prod_{i=1}^{n}\left(1-\left(a_{i 1}, a_{i 2}, a_{i 3}\right)\right) \\
& =\left(1-\prod_{i=1}^{n}\left(1-a_{i 1}\right), 1-\prod_{i=1}^{n}\left(1-a_{i 2}\right), 1-\prod_{i=1}^{n}\left(1-a_{i 3}\right),\right)=\tilde{p}_{y}=\left(a_{y 1}, a_{y 2}, a_{y 3}\right), \text { say }
\end{aligned}
$$

In a similar manner if $p_{i}$ 's are trapezoidal fuzzy numbers that is $p_{i}=\left(a_{i 1}, a_{i 2}, a_{i 3}, a_{i 4}\right)$, then

$$
\begin{gathered}
\tilde{p}_{y}=\operatorname{ANF}\left(\tilde{p}_{1}, \tilde{p}_{2}, \ldots \ldots \ldots \ldots \ldots . . . \tilde{p}_{n}\right)=\left(\prod_{i=1}^{n} a_{i}, \prod_{i=1}^{n} a_{i}, \prod_{i=1}^{n} a_{i}, \prod_{i=1}^{n} a_{i}\right)=\left(a_{y 1}, a_{y 2}, a_{y 3}, a_{y 4}\right), \text { say } \\
\tilde{p}_{y}=\operatorname{ORF}\left(\tilde{p}_{1}, \tilde{p}_{2}, \ldots \ldots \ldots \ldots \ldots . . . \tilde{p}_{n}\right)=\left(1-\prod_{i=1}^{n}\left(1-a_{i 1}\right), 1-\prod_{i=1}^{n}\left(1-a_{i 2}\right), 1-\prod_{i=1}^{n}\left(1-a_{i 3}\right), \text { and } 1-\right. \\
\left.\prod_{i=1}^{n}\left(1-a_{i 4}\right)\right)=\left(a_{y 1}, a_{y 2}, a_{y 3}, a_{y 4}\right),
\end{gathered}
$$

\section{APPROXIMATIONS OF FUZZY NUMBERS:}

Once the data about the occurrence of the basic events is obtained we can approximate a fuzzy number for each basic event using any method for construction of membership function. The membership function of the basic event may generally be any function but usually these are considered to represent triangular or trapezoidal fuzzy numbers.

Suppose that the data about the occurrence of the basic events are provided to $n$ experts and they are asked to assign a possibility function (fuzzy number) 
corresponding to it. Let $A_{i}$ denotes the triangular fuzzy number formulated by the expert $(i=1,2 \ldots n$.) and is given as below:

$$
A_{i}=\left(a_{i}-c_{i} . a_{i}, a_{i}+c_{i}\right)
$$

Let $B=(b-d, b, b+d)$ denote a number that tunes with the judgment of all experts. All $A_{i}$ 's are used to determine the values of the parameters $b$ and $d$. For this we find $B-A^{i}$, which is again a triangular fuzzy number. It is well known that smaller triangular fuzzy number will result in the better approximation for $B$. The height of $B-A^{i}$ cannot be reduced, since it must always be one. Therefore our measure depends on the length of base line of the triangle. For this we suppose, let

$$
S=\sum\left[2\left(d-c_{i}\right)\right]^{2}
$$

Then $S$ will achieve its minimum if

$$
d=\frac{1}{n} \sum_{i=1}^{n} c_{i}
$$

Further if we want to determine the parameter $b$, we suppose

$$
\mathrm{D}=\max _{1 \leq i \leq n}\left|b-a_{i}\right|
$$

Then $\mathrm{D}$ will be minimum for,

$$
b=\frac{\min _{1 \leq i \leq n} a_{i}+\max _{1 \leq i \leq n} a_{i}}{2}
$$

This approach can also be applied to deal with trapezoidal fuzzy numbers. Let $A_{i}=\left(a_{i}-c_{i}, a_{i}, a_{i}^{\prime}, a_{i}^{\prime}+c_{i}\right), i=1,2 \ldots \mathrm{n}$. are $\mathrm{n}$ trapezoidal fuzzy numbers assigned to the failure possibility of a basic event. Then a trapezoidal fuzzy number $A_{i}=\left(b_{i}-d_{i}, b_{i}, b_{i}^{\prime}, b_{i}^{\prime}+d_{i}\right)$ which is the fuzzy number that fits with all experts' decision can be given by the following expressions

$$
\begin{gathered}
d=\frac{1}{n} \sum_{i=1}^{n} c_{i}, \\
b=\frac{\min _{1 \leq i \leq n} a_{i}^{\prime}+\max _{1 \leq i \leq n} a_{i}^{\prime}}{2} \quad \text { and } \quad b^{\prime}=\frac{\min _{1 \leq i \leq n} a_{i}+\max _{1 \leq i \leq n} a_{i}}{2}
\end{gathered}
$$

\section{SOLUTION OF THE MODEL:}

By using algebra of logics, equation (1) may be written as:

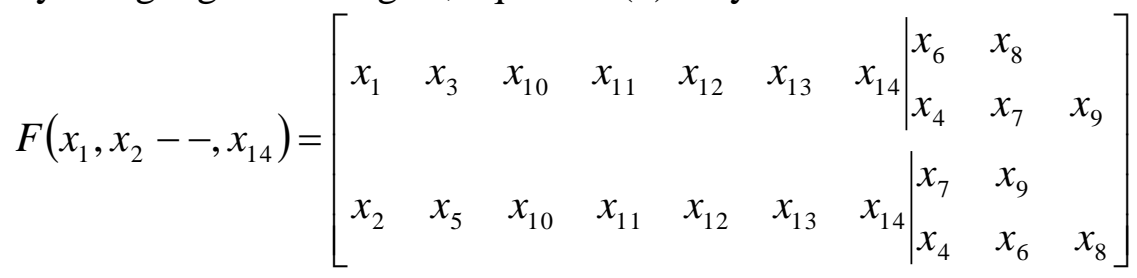




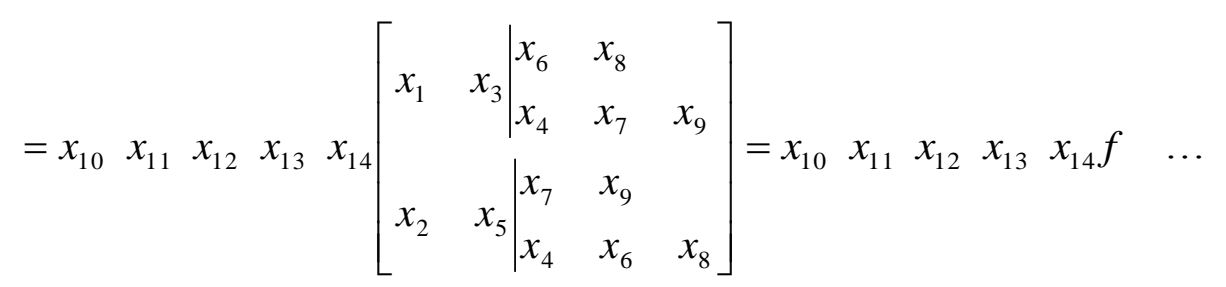

where,

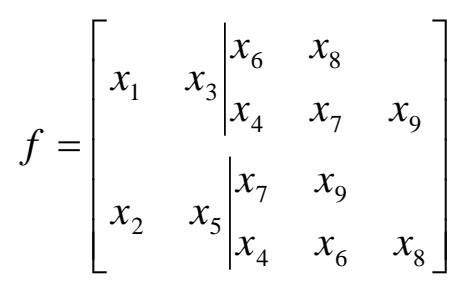

In equation (3), five arguments $\left(x_{4}, x_{6}, x_{7}, x_{8}, x_{9}\right)$ are entering twice. Therefore, we can take expansion from any one of them. Let we choose expansion from $x_{6}$,

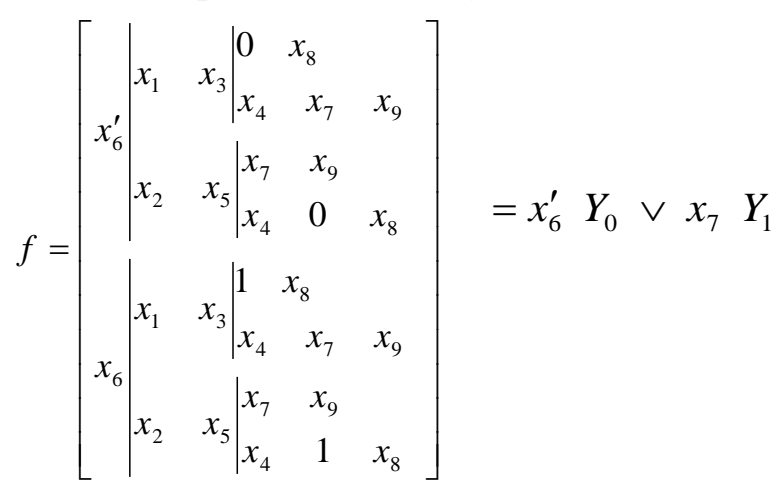

where,

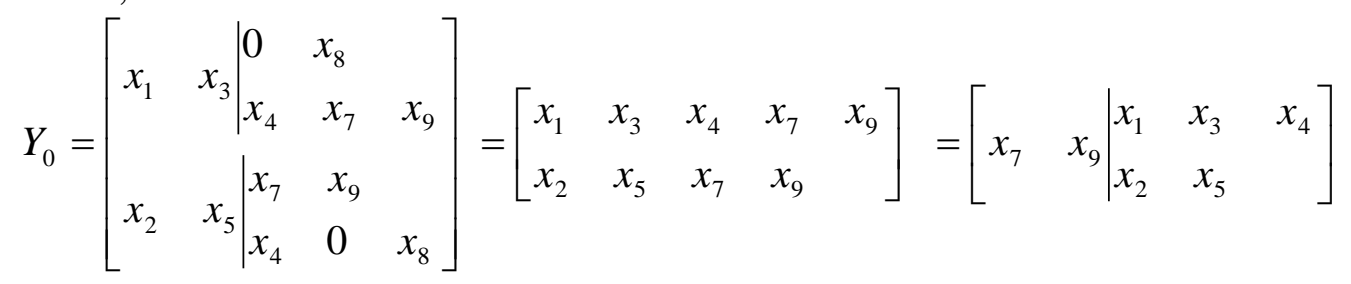

and

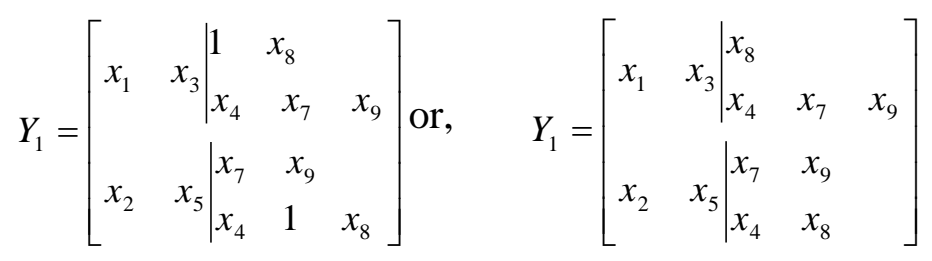

All the arguments in equation (5) appear only once, hence $Y_{0}$ is non -iterated but the arguments $\left(x_{4}, x_{7}, x_{8}, x_{9}\right)$ enter twice in $Y_{1}$, therefore any one of them may be taken to perform further this expansion. Let us conveniently choose $x_{7}$ and do expansion as follows: 


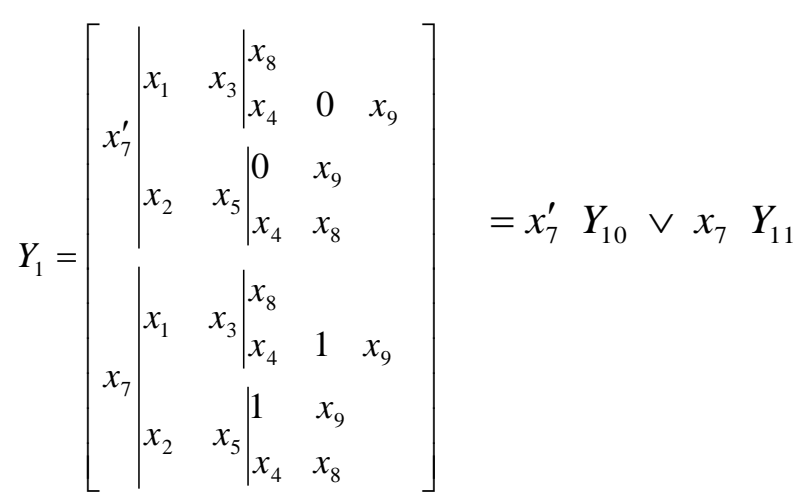

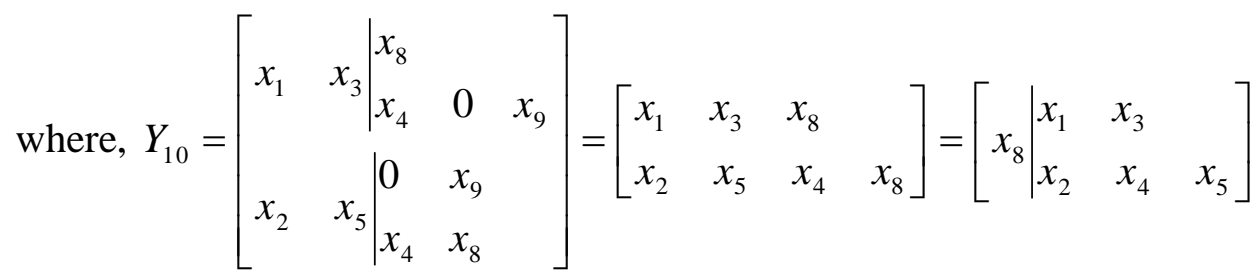

and

$$
Y_{11}=\left[\begin{array}{ll|l|l}
x_{1} & x_{3} & x_{8} & \\
x_{4} & x_{9} \\
x_{2} & \left.x_{5} \mid \begin{array}{ll}
x_{9} & \\
x_{4} & x_{8}
\end{array}\right]
\end{array}\right]
$$

In equation (8) all the arguments appear once and so $Y_{10}$ is non -iterated. In equation (9) the arguments $\left(x_{4}, x_{8}, x_{9}\right)$ appear twice therefore we may take any one of them for further proceedings. Let us choose $x_{8}$ for the next expansion. Thus,

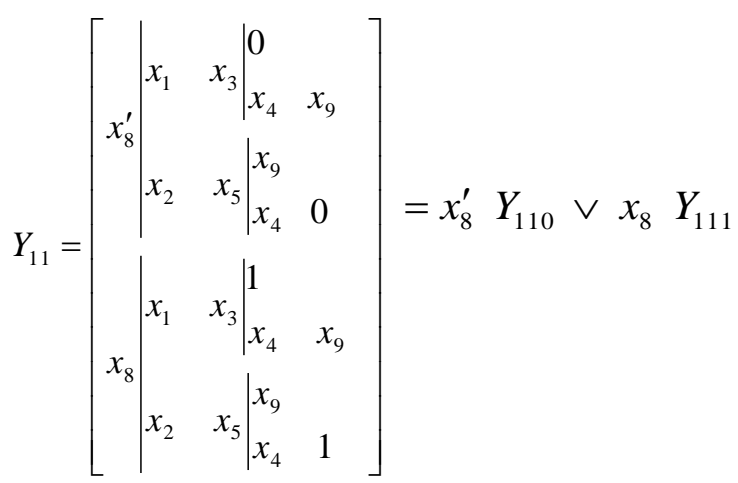

where,

$$
Y_{110}=\left[\begin{array}{ll|ll}
x_{1} & x_{3} & 0 \\
x_{4} & x_{9} \\
x_{2} & x_{5} & x_{9} & \\
x_{4} & 0
\end{array}\right]=\left[\begin{array}{llll}
x_{1} & x_{3} & x_{4} & x_{9} \\
x_{2} & x_{5} & x_{9} &
\end{array}\right]=\left[x_{9} \mid \begin{array}{lll}
x_{1} & x_{3} & x_{4} \\
x_{2} & x_{5}
\end{array}\right]
$$


and

$$
Y_{111}=\left[\begin{array}{ll|ll}
x_{1} & x_{3} & \\
x_{4} & x_{9} \\
x_{2} & x_{5} & x_{9} & \\
x_{4} & 1
\end{array}\right]=\left[\begin{array}{ll|l}
x_{1} & x_{3} \\
x_{2} & x_{5} & x_{9} \\
x_{4}
\end{array}\right]
$$

In equations (11) and (12), all the arguments appear once, hence both functions $Y_{110}$ and $Y_{111}$ are non-iterated and are not subjected to further expansion. Hence equation (4) gives, by using equations (5) through (12):

$$
f=\left[\begin{array}{llll}
x_{6}^{\prime} & Y_{0} & & \\
& x_{7}^{\prime} & Y_{10} & \\
x_{6} & & x_{8}^{\prime} & Y_{110} \\
& x_{7} & x_{8} & Y_{111}
\end{array}\right]=\left[\begin{array}{cccc}
x_{6}^{\prime} & Y_{0} & & \\
x_{6} & x_{7}^{\prime} & Y_{10} & \\
x_{6} & x_{7} & x_{8}^{\prime} & Y_{110} \\
x_{6} & x_{7} & x_{8} & Y_{111}
\end{array}\right]=\left[\begin{array}{l}
H_{1} \mid Y_{0} \\
H_{2} \mid Y_{10} \\
H_{3} \mid Y_{110} \\
H_{4} \mid Y_{111}
\end{array}\right]
$$

where, $H_{1}=x_{6}^{\prime}, H_{2}=x_{6} x_{7}^{\prime}, H_{3}=x_{6} x_{7} x_{8}^{\prime}$ and $H_{4}=x_{6} x_{7} x_{8}$.

Clearly $H_{1}, H_{2}, H_{3}$ and $H_{4}$ are pair-wise disjoint.

$$
\therefore P_{r}(f=1)=\sum_{i=1}^{4} P_{r}\left(H_{i}\right) \cdot P_{r}\left(\frac{f}{H_{i}}\right)
$$

or, $P_{r}(f=1)=P_{r}\left(x_{6}^{\prime}\right) \cdot P_{r}\left(z_{1}\right)+P_{r}\left(x_{6} x_{7}^{\prime}\right) \cdot P_{r}\left(z_{2}\right)+P_{r}\left(x_{6} x_{7} x_{8}^{\prime}\right) \cdot P_{r}\left(z_{3}\right)$

$$
+P_{r}\left(x_{6} x_{7} x_{8}\right) \cdot P_{r}\left(z_{4}\right)
$$

Where, $z_{1}=Y_{0}, z_{2}=Y_{10}, z_{3}=Y_{110}$ and $z_{4}=Y_{111}$ and the events $H_{i}(\forall i=1,2,3,4)$ form a complete group of incompatible hypothesis. Then $P_{r}\left(f / H_{i}\right)$ form the conditional probability of a good state of the system for each hypothesis.

Now, if $R_{i}$ be the reliability corresponding to component state $x_{i}$, then equation (14) gives:

$$
\begin{aligned}
& P_{r}(f=1)=\left(1-R_{6}\right) R_{7} R_{9}\left\{1-\left(1-R_{1} R_{3} R_{4}\right)\left(1-R_{2} R_{5}\right)\right\} \\
& +R_{6}\left(1-R_{7}\right) R_{8}\left\{1-\left(1-R_{1} R_{3}\right)\left(1-R_{2} R_{4} R_{5}\right)\right\} \\
& +R_{6} R_{7}\left(1-R_{8}\right) R_{9}\left\{1-\left(1-R_{1} R_{3} R_{4}\right)\left(1-R_{2} R_{5}\right)\right\} \\
& +R_{6} R_{7} R_{8}\left[1-\left(1-R_{1} R_{3}\right) \times\left\{1-R_{2} R_{5}\left(1-\left(1-R_{9}\right)\left(1-R_{4}\right)\right)\right\}\right]
\end{aligned}
$$

Finally, the probability of successful operation, i.e., reliability of the considered system is given by:

$$
\begin{aligned}
R_{S}=P_{r}(F=1) & =P_{r}\left(x_{10} x_{11} x_{12} x_{13} x_{14}\right) \cdot P_{r}(f=1) \\
& =R_{10} R_{11} R_{12} R_{13} R_{14}\left[R_{2} R_{5} R_{7} R_{9}+R_{1} R_{3} R_{4} R_{7} R_{9}+R_{1} R_{3} R_{6} R_{8}+R_{2} R_{4} R_{5} R_{6} R_{8}\right. \\
& +R_{1} R_{2} R_{3} R_{4} R_{5} R_{6} R_{7} R_{8} R_{9}+R_{1} R_{2} R_{3} R_{4} R_{5} R_{6} R_{7} R_{8} R_{9}-R_{1} R_{2} R_{3} R_{4} R_{5} R_{7} R_{9} \\
& -R_{1} R_{2} R_{3} R_{4} R_{5} R_{6} R_{8}-R_{1} R_{3} R_{4} R_{6} R_{7} R_{8} R_{9}-R_{2} R_{4} R_{5} R_{6} R_{7} R_{8} R_{9}
\end{aligned}
$$




$$
\left.-R_{1} R_{2} R_{3} R_{5} R_{6} R_{7} R_{8} R_{9}\right]
$$

Particular Cases:

Case I: When reliability of each component of system is $R$ :

In this case, equation (17) yields:

$$
\begin{array}{r}
R_{S}=R^{5}\left[2 R^{4}+4 R^{5}-2 R^{7}-R^{8}+2 R^{9}\right] \\
=R^{9}\left[2+2 R-4 R^{3}-R^{4}+2 R^{5}\right]
\end{array}
$$

In case of fuzzy reliability, when reliability of each component is $\tilde{R}$

$$
\begin{array}{r}
\widetilde{R}_{S} \cong \widetilde{R}^{5}\left[2 \widetilde{R}^{4}+4 \widetilde{R}^{5}-2 \widetilde{R}^{7}-\widetilde{R}^{8}+2 \widetilde{R}^{9}\right] \\
\cong \widetilde{R}^{9}\left[2+2 \widetilde{R}-4 \widetilde{R}^{3}-\widetilde{R}^{4}+2 \widetilde{R}^{5}\right]
\end{array}
$$

\section{Case II: When failure rates follow weibull distribution:}

In this case, the reliability of considered system is given by:

$$
\mathrm{R}_{\mathrm{SW}}(t)=\sum_{i=1}^{6} \exp .\left\{-a_{i} t^{\alpha}\right\}-\sum_{j=1}^{5} \exp \cdot\left\{-b_{j} t^{\alpha}\right\}
$$

Where, $\alpha$ is a real positive parameter and

$$
\begin{aligned}
& a_{1}=c+\lambda_{2}+\lambda_{5}+\lambda_{7}+\lambda_{9} \\
& a_{2}=c+\lambda_{1}+\lambda_{3}+\lambda_{4}+\lambda_{7}+\lambda_{9} \\
& a_{3}=c+\lambda_{1}+\lambda_{3}+\lambda_{6}+\lambda_{8} \\
& a_{4}=c+\lambda_{2}+\lambda_{4}+\lambda_{5}+\lambda_{6}+\lambda_{8} \\
& b_{1}=c+\lambda_{1}+\lambda_{2}+\lambda_{3}+\lambda_{4}+\lambda_{5}+\lambda_{7}+\lambda_{9} \\
& b_{2}=c+\lambda_{1}+\lambda_{2}+\lambda_{3}+\lambda_{4}+\lambda_{5}+\lambda_{6}+\lambda_{8} \\
& b_{3}=c+\lambda_{1}+\lambda_{3}+\lambda_{4}+\lambda_{6}+\lambda_{7}+\lambda_{8}+\lambda_{9} \\
& b_{4}=c+\lambda_{2}+\lambda_{4}+\lambda_{5}+\lambda_{6}+\lambda_{7}+\lambda_{8}+\lambda_{9} \\
& \text { and } c=\lambda_{10}+\lambda_{11}+\lambda_{12}+\lambda_{13}+\lambda_{14}
\end{aligned}
$$

Where $\lambda_{i}$ be the failure rate corresponding to system state $x_{i}$.

In case of fuzzy reliability

$$
\tilde{\mathrm{R}}_{\mathrm{fSW}}(t)=\sum_{i=1}^{6} \exp \cdot\left\{-\tilde{\lambda} a_{i} t^{\alpha}\right\}-\sum_{j=1}^{5} \exp \cdot\left\{-\tilde{\mu} b_{j} t^{\alpha}\right\}
$$

\section{Case III: When failure rates follow exponential distribution:}

Exponential time distribution is the particular case of weibull time distribution for $\alpha=1$ and is very useful in numerous practical problems. Therefore the reliability function for the whole system at time instant ' $t$ ', is given by:

$$
\begin{aligned}
\mathrm{R}_{\mathrm{SE}}(t) & =\sum_{i=1}^{6} \exp \cdot\left\{-a_{i} t\right\}-\sum_{j=1}^{5} \exp \cdot\left\{-b_{j} t\right\} \\
\tilde{\mathrm{R}}_{\mathrm{fSE}}(t) & =\sum_{i=1}^{6} \exp \cdot\left\{-\tilde{\lambda} a_{i} t\right\}-\sum_{j=1}^{5} \exp \cdot\left\{-\tilde{\mu} b_{j} t\right\}
\end{aligned}
$$


where $a_{i}^{\prime} s, b_{j}^{\prime} s, \tilde{\lambda}$ and $\tilde{\mu}$ have been mentioned earlier.

Also, in this case, an important reliability parameter M.T.T.F, is given by

$$
\text { M.T.T.F. }=\int_{0}^{\infty} R_{S E}(t) d t=\sum_{i=1}^{6}\left(\frac{1}{a_{i}}\right)-\sum_{j=1}^{5}\left(\frac{1}{b_{j}}\right)
$$

And the parameter mean time to fuzzy failure (M.T.T.F.F.) is given by

$$
M . T . T . F . F .=\int_{0}^{\infty} \tilde{R}_{f S E}(t) d t=\sum_{i=1}^{6}\left(\frac{1}{\tilde{\lambda} a_{i}}\right)-\sum_{j=1}^{5}\left(\frac{1}{\tilde{\mu} b_{j}}\right)
$$

\section{NUMERICAL COMPUTATION:}

For a numerical computation, let us consider the values:

(i) $\lambda_{i}(i=1,2,--14)=\lambda=0.001, \alpha=2$ and $\mathrm{t}=0,1--2$. Using these values in equation (19), we compute the table -1 .

(ii) $\lambda_{i}(i=1,2,--14)=\lambda=0.001$ and $\mathrm{t}=0,1,2-\cdots$. Using these values in equation (20), we compute the table-1.

(iii) Putting $\lambda_{i}(i=1,2,--14)=\lambda=0.001,0.002--0.01$ in equation (21), we compute table-2.

For a fuzzy aspect the numerical computation will be considered by fuzzification and then defuzzification of the values which will cover up the maximum uncertainties, let us consider the values:

(ia) $\lambda_{i}(i=1,2,--14)=\lambda=0.00112, \alpha=0,1,2$ and $\mathrm{t}=0,1,2--$. Using these values in equation (19), we compute the table -1 .

(iib) $\lambda_{i}(i=1,2,-14)=\lambda=0.00112$ and $\mathrm{t}=0,1,2$-. Using these values in equation (20), we compute the table- 1 .

(iiic) Putting $\lambda_{i}(i=1,2,--14)=\lambda=0.00112,0.0012--0.01$ in equation (21), we compute table-2.

\section{Table-1}

\begin{tabular}{|c|c|c|c|c|}
\hline $\mathbf{T}$ & $R_{S W}(t)$ & $\tilde{R}_{F S W}(t)$ & $R_{S E}(t)$ & $\tilde{R}_{F S E}(t)$ \\
\hline 0 & 1 & 1.000000 & 1 & 1.000000 \\
\hline 1 & 0.995005 & 0.999987 & 0.995005 & 0.998456 \\
\hline 2 & 0.980074 & 0.998653 & 0.990018 & 0.996754 \\
\hline 3 & 0.955384 & 0.972658 & 0.985041 & 0.991567 \\
\hline 4 & 0.921259 & 0.943218 & 0.980074 & 0.989876 \\
\hline 5 & 0.878210 & 0.901254 & 0.975116 & 0.982345 \\
\hline 6 & 0.826981 & 0.882345 & 0.970168 & 0.978654 \\
\hline 7 & 0.768586 & 0.824561 & 0.965230 & 0.968923 \\
\hline 8 & 0.704330 & 0.796574 & 0.960302 & 0.961715 \\
\hline 9 & 0.635794 & 0.728756 & 0.955384 & 0.960234 \\
\hline 10 & 0.564783 & 0.698765 & 0.950476 & 0.954321 \\
\hline
\end{tabular}


Table-2

\begin{tabular}{|c|c|c|c|}
\hline \multicolumn{1}{|l|}{$\lambda$} & M.T.T.F. & $\tilde{\lambda}$ & M.T.T.F.F. \\
\hline 0 & $\infty$ & 0 & $\infty$ \\
\hline 0.001 & 154.8231 & 0.00112 & 162.25806 \\
\hline 0.002 & 77.41148 & 0.00123 & 86.39024 \\
\hline 0.003 & 51.60765 & 0.00168 & 69.60784 \\
\hline 0.004 & 38.70574 & 0.00187 & 46.39344 \\
\hline 0.005 & 30.96459 & 0.00213 & 34.08451 \\
\hline 0.006 & 25.80383 & 0.00289 & 32.34568 \\
\hline 0.007 & 22.11756 & 0.00312 & 24.98901 \\
\hline 0.008 & 19.35873 & 0.00384 & 21.90099 \\
\hline 0.009 & 17.20255 & 0.00412 & 19.09009 \\
\hline 0.010 & 15.48230 & 0.00488 & 18.26463 \\
\hline
\end{tabular}

\section{DISCUSSION:}

Analysis of table-1 reveals that value of reliability function decreases rapidly, in case, failures follow Weibull time distribution but it decreases merely in constant manner for exponential time distribution but the fuzzy reliability gracefully decreases in respect of the above distribution. Therefore, reliability and the fuzzy reliability functions remains better in case of exponential time distribution. But the Weibull distribution covers more uncertainties with respect to more parameters. A critical examination of table- 2 concludes that M.T.T.F. decreases catastrophically for the lower values of failure rate $\lambda$ but it decreases smoothly for higher values of $\lambda$ while the M.T.T.F.F. deceases more realistically than the M.T.T.F. for the lower values of the failure rates. On the basis of the above theories we can conclude that the classical set theoretic approach does not seem very effective to cover up most of the uncertainties occurred in the data and various statistical methods used for failure rate estimation and reliability evaluation of a system. Here, in this research paper we have introduced a new approach for failure rate estimation based on fuzzy set theory, which is more realistic approach in case of system behaviour. Also the degradation of the system is considered to be fuzzy in nature rather than crisp i.e. various system states have been taken between a fully working and fully failed state to evaluate the reliability of the system. The classical reliability of the system, which is a particular case of fuzzy reliability, has also been evaluated.

\section{REFERENCES}

[1]. L. A. Zadeh, "Fuzzy Sets," Information and Control, Vol. 8, No. 3, 1965, pp. 338-353. doi:10.1016/S0019-9958(65)90241-X 
[2]. Chin Torng Lin \& Mao- Jun J. Wang (1997), "Hybrid fault tree analysis using fuzzy sets", Reliability Engineering and system safety 58 205-213

[3]. C. Y. Wen, K. Y. Cai, and M. L. Zhang, "Posbist reliability behaviour of typical system with two type of failures", Fuzzy Sets and Systems, 43, 17-32, 1991.

[4]. M.K. Sharma and D. pandey, Fuzzy Reliability and Fuzzy Availability of a Three Unit Degradable System, International J. of Math. Sci. \& Engg. Appls. (IJMESA), ISSN 0973-9424, Vol.3 No. II (2009), pp.199-213.

[5]. M. Oussalah and M. Newby, "Analysis of serial-parallel systems in the framework of Fuzzy/possibility approach." Reliability Engineering and system safety. Vol. 79 (2003); pp. 353-368

[6]. Zadeh L.A., "Probability theory and fuzzy logic are complementary rather than Competitive." Technometrices. 37 (3) (1995); pp. 271-279

[7]. L.A.Zadeh, Fuzzy sets as a basis for a theory of possibility, Fuzzy, sets and systems 1 (1978) 3-28.

[8]. Zadeh L.A., "Fuzzy logic." IEEE Transactions on Computer, (21) (1988); pp:83-93

[9]. Cai K.Y. and Wen C.Y., "Fuzzy variables as a basis for a theory of fuzzy reliability in the possibility context" in Fuzzy sets and systems, 42, (1991a); pp. 145-172

[10]. Dhillon, B. S.; Natesan, J.: "Stochastic Analysis of Outdoor Power System in Fluctuating Environment", Microelectronic Reliab. , Vol.23, pp 867 - 887, 1983.

[11]. Gupta, P.P.; Sharma, R.K.: "Cost Analysis of Three-state Standby Redundant Electronic Equipment", Microelectronic Reliab. , Vol. 25, pp 1029-1033, 1985.

[12]. Jain, S.P.; Gopal, K.: "A Heuristic Method of Link Reliability Assignment for Maximal Reliability”, Microelectronic Reliab., Vol. 30, pp 673 - 679, 1990.

[13]. Jen - Shyan, W.; Rong - Jaye, C.: "Efficient Algorithm for Reliability of a Circular Consecutive k-out-of-n: F System", IEEE TR on Reliability, Vol. 55, issue - 1, pp 163-164, 2006.

[14]. Kuo, W; Prasad, V.R.; Tillman, F.A. and Hwang, C.L: "Fundamental and Applications of Reliability Optimization", Cambridge University Press, Cambridge, 2000.

[15]. Nagraja, H.N.; Kannan, N.; Krishnan, N.B.: "Reliability", Springer Publication, 2004 
[16]. Sharma, Deepankar : "Cost analysis, availability and MTTF of a complex system with the concept of waiting and human error", Bulletin of pure and applied sciences, Vol. 21 E, No. 2, pp 553-559 (2002).

[17]. Singh, J.; Goyal, P.: "Availability analysis of a standby complex system having imperfect switch over device", Microelectron. Reliab. 35, pp 285-288 (1996)

[18]. Vanderhaegen, F.; Jouglet, D.; Piechowiak, S.: "Human Reliability Analysis of Co-operative Redundancy to Support Diagnosis", IEEE TR. on Reliability, Vol. 53, issue-4, pp 458-464, 2004

[19]. Zhimin, He; Han, T.L.; Eng., H.O.: "A Probabilistic Approach to Evaluate the Reliability of Piezoelectric Micro-Actuators", IEEE TR. on Reliability, Vol. 54, issue-1, pp 83-91, 2005. 